\title{
Network Security Based on Component for Information Network
}

\author{
A. Aruljothi ${ }^{1}$, N. Prabhu ${ }^{2}$ and N. M. Mallika ${ }^{3}$ \\ ${ }^{1 \& 2}$ Assistant Professor, Department of Computer Science, Gobi Arts \& Science College, Tamil Nadu, India \\ ${ }^{3}$ Dean \& Assistant Professor, Department of Computer Science, Sri Vasavi College, Tamil Nadu, India \\ E-Mail: tamil.aruljothi@gmail.com, gascprabhu@gmail.com,nm.mallika@gmail.com
}

\begin{abstract}
It is a difficult building to ensure the security of data arrange, so it is important to consider out a new security control design and model in the viewpoint of foundational control. As indicated by the attributes and security requests of data net, another security control viewpoint and its engineering dependent on parts is proposed, the security control framework is made-up, and insights regarding the structure of control system, its different kinds, and capacities and spread methods of security control parts are presented, at that point the highlights of the control framework are outlined at last.
\end{abstract}

Keywords: Component, Info-Net, Trust Domain, Security, Security Control, Architecture

\section{INTRODUCTION}

In the event that the PC organize is viewed as a complex gigantic framework, it's made out of conceptual elements and the relations among the substances. The element is indicated the practical unit having free personality, for example, PC hardware, correspondence hardware and the procedure or administration in the PC working framework, and so forth. In "Dark Reading: Automating Breach Detection For The Way Security Professionals Think” [1]. the relations among the substances are portrayed by correspondence joins So as to ensure the security of PC arranges, the most vital is to ensure the security of key elements and key correspondence joins dependent on security necessities. Accordingly, "Stallings and Brown, Computer Security: Principles and Practice” [2]. customary organize security arrangements utilize a wide range of security innovations and create different security items, for precedent, encryption programming, firewall, interruption discovery framework (IDS), Virtual Private Network (VPN, etc. These items can protect related systems and types of gear, yet arrange security is a procedure and isn't straightforward accumulation of security items [2]. In "Stamp, Information Security: Principles and Practice"[3] The system security dangers and necessities are persistently evolving. It's exceptionally hard to entirely examine framework security chances and give adaptable security benefit appropriate for the changing prerequisites from the holy messenger of framework control if dispersive security items absence of bound together the executives and association are just utilized. So security items ought to acknowledge work specialization and part frame/s and be equivalent to other system elements (NEs) utilizing uniform interfaces of security control and connection. Secure PC systems can be looked on as a security compartment, which can adjust and control oneself, including different security control segments (SCCs), arrange elements and correspondence joins, showed as Fig. 1.

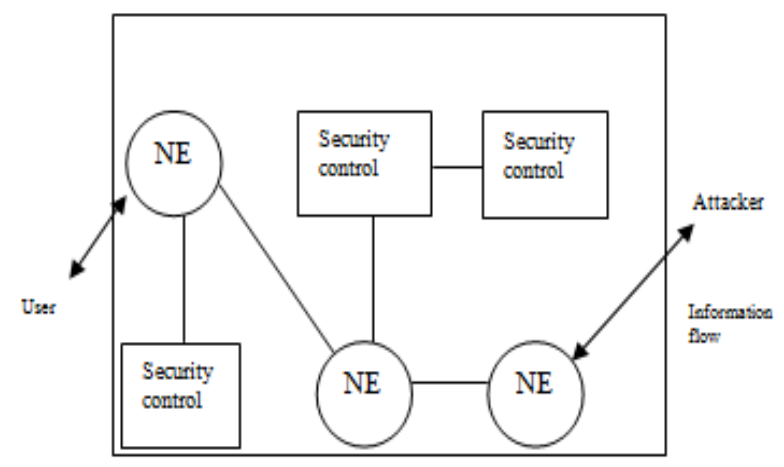

Fig. 1 Abstract structure of secure computer network

They interact with each other and security of information storage, transmission; processing and utilization can be realized with the support of network security infrastructure such as PKI (Public Key Infrastructure), KMI (Key Management Infrastructure) or PMI (Privilege Management Infrastructure) "Goodrich and Tamassia, Introduction to Computer Security”[4]. The security control architecture (SCA) of info-net based on components is proposed using the principles and methods of network cybernetics. The security control system is realized, the management methods of security infrastructure and security control components, the types, functions and propagation modes of security control components are also elaborated.

\section{SECURITY CONTROL ARCHITECTURE OF INFONET}

Data net security control system dependent on parts is made out of security clients, security administrations, the executives system of security control (counting security framework the executives (SIM) and the administration of SCCs), controlled organize objects (counting system substances and correspondence joins), security control segments and security control framework. So as to adjust the attributes of appropriated the board of system elements, the security control framework is utilized. It gives the premise to setting up and holding together the trust relations among system substances and overseeing and confirming the substance characters "Zhang Haixia, Lian Yifeng, Su Purui, and Feng Dengguo" [5]. Also, its LDAP 
(Lightweight Directory Access Protocol) server can give inquiry administration to the substance authentications, approval authentications and security control segments. CA (Certificate Expert) in PKI makes the character for each authentic arrange substance and security control segment and gives validation benefit.

Clients make security strategies dependent on security chance evaluation and security necessities and propose security benefit necessities. These prerequisites will be acknowledged by the control stubs introduced in system substances. The control stub compares with the security operator of a system substance, which is a segment of some basic program and can acknowledge clients' security benefit prerequisites. The control stub can collaborate with security control parts and security control framework, seek, introduce and refresh relating security control parts as per the security benefit asks for and validate control segments. The security framework the executive's framework assumes liability for the executives of CA and testaments. The administration framework of security control segments assumes liability for the enrollment, refresh and log-out of security control segments. The relations among the security control foundation. The constituent parts collaborate with

1. Management Protocol of Security Service Prerequisites: Users collaborate with the control stubs in system substances by the convention. It includes, adjusts and erases the security benefit prerequisites of each system element.

2. Communication Protocol between Network Entities and Security Control Components: Network substances interface with security control segments introduced in substances by the convention and give relating security administrations.

3. Management Protocol of Security Control Foundation: The administration arrangement of security framework oversees CA and testaments by the convention, counting creation, refresh, cancellation of CA and creation, refresh what's more, log-out of the personalities (endorsements) of system substances.

4. Management of Security Control Components: The board arrangement of security control segments oversees security control parts, including enrollment, refresh and log-out of parts and the computerized mark of segments.

5. Communication Protocol between Security Control Parts: The linkage among segments can be figured it out through standard capacity call interfaces.

6. Communication Protocol between Network Entities and Security Control Infrastructure: The characters of system substances and the validness and trustworthiness of system control parts can be confirmed by the convention.

7. Communication Protocol among Network Entities: Through the connection between system substances and control stubs, the engendering and refresh of security control segments can be executed.

\section{A. Control Management Framework}

Information net security control the board structure dependent on parts incorporates the administration of security foundation furthermore, security controls parts. So as to adjust the disseminated attributes of system elements, the security control framework utilizing PKI is in charge of declaration issue, inquiry, validation, refresh and log-out for system elements. As to PKI, the sorting out structure of CA decides its effectiveness and security. There are two primary sorts of CA arranging structure of customary PKI. One is dispersive CA arranging structure, whose delegate is the declaration framework utilized by PGP (Pretty Good Privacy). There isn't strict progressive relations, neither the root CA accepted by all CA. Authentications can be issued commonly. The other is incorporated CA arranging structure, whose delegate is the declaration framework utilized by PEM (Privacy Enhanced Mail).

The authentication is issued from root CA to the hypo-level and from the hypo level to the following dimension. These two sorts of CA sorting out structure have one's very own excellencies and weaknesses. Unified structure is with high security. Since each CA has sole declaration trust chain with the root CA, the unwavering quality of each declaration can be actually checked. Its unwavering quality isn't solid, when some CA of the testament chain endures hurt, CA also; the dependability of all CA and testaments administered by it will additionally be hurt. Dispersive structure is inverse to the unified. Each CA can confide in numerous other CA, so the trust chain among CA isn't sole.

Hurting parts of CA won't cause the breakdown of the entire PKI. Each CA confides in other CA as indicated by a few standards. These CA establishes the trust fields where they trust each other in neighborhoods they issue authentications commonly.

Each CA can believe other CA in the trust field, and in the meantime each CA has its predominant CA who can guarantee its unwavering quality and is overseen by sole root CA. Demonstrated by the hypothesis and practice and contrasted and PEM, the CA sorting out structure related with trust fields doesn't diminish the validity of declarations. It expands the unwavering quality of PKI and the proficiency of declaration confirmation and refresh. It's reasonable for giving verification and advanced approval benefit for disseminated system elements and security control segments. Distributed security control system uses the CA organizing structure based on trust fields, illustrated as Fig. 2. 


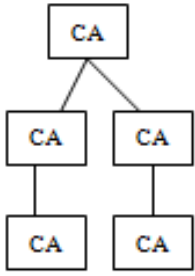

Centralized structure

Fig. 2 The organizing structure of CA

The administration of SCC utilizes the strategy for dispersive creation, concentrated enlistment and question. For whatever length of time that they conform to brought together capacity interface standard of SCC, each substance can make SCC and signs by one's very own private key to secure its trustworthiness. At that point it conveys to the administration framework of SCC to assess the similarity, work agreement and security.

In the wake of passing the assessment, the segment will be doled out a select personality. The character is marked by the private key of the administration framework and appropriated to LDAP servers for the utilization of other system substances. The work interface standard of security control parts controls the characteristics and techniques for segment depiction, counting part names, sources, advanced mark and work interfaces for the comfort of the association among segments and NEs.

\section{SECURITY CONTROL SYSTEM OF INFO-NET}

In the security control engineering dependent on parts, we can fabricate a circulated security control arrangement of information net. It is made out of staggered security control focus and security control stages introduced in system substances,

A. Control User Operations: It allows that certified clients can work terminals after confirmation and utilizations the validation techniques for double or different factors so as to enhance the security of client signing on. This can help dispose of the activities surpassing one's very own position of lawful clients and unlawful activities of illicit clients. The activities of qualified clients and task endeavors of illicit clients are recorded in term of the prerequisites of the security control focus to guarantee task security of the terminals.
B. Control Program Execution: It controls the execution of working framework and applications on terminals. Through the control of the security control focus, blemishes are found and patched. The honesty of projects and execution pictures ought to be verified and just approved projects can be executed by the approval while PC infections also; different malevolent projects are avoided so as to guarantee execution security of the terminals. Other than fringe gear interfaces ought to be controlled and it's as it were allowed to powerfully stack or introduce drivers passing uprightness confirmation.

\section{CONCLUSION}

It proposes a security control design dependent on security control necessities of data net. In the security control framework dependent on segments of data net, organize elements are the makers, as well as the buyers of security control parts. SCC has the qualities of work specialization, interface institutionalization, reproducibility, refresh and propagability. The security control the board system plans SCCs consistently and it makes a difference understand the dispersed control on interest of system security. Regardless of whether the sorting out structure of CA or the engendering strategy for segments are equivalent and adjusted, which builds the security control proficiency and diminishes the control multifaceted nature. In the meantime, they additionally enhance the adaptability and dependability of security control. The control design and thought is particularly appropriate for security control of the dynamic, portable and private system, for example, space data arrange, strategic C3I, etc.

\section{REFERENCES}

[1] "Dark Reading: Automating Breach Detection for the Way Security Professionals Think”, October 1, 2015.

[2] Stallings, and Brown, Computer Security: Principles and Practice, 3/e, Prentice Zhang Haixia, Lian Yifeng, Su Purui, and Feng Dengguo Hall, 2014.

[3] Stamp, Information Security: Principles and Practice, 2/e, Wiley, 2011.

[4] Goodrich and Tamassia, Introduction to Computer Security AddisonWesley, 2010.

[5] Zhang Haixia, Lian Yifeng, Su Purui, and Feng Dengguo, "Securitystate-Region-Based Model of Network Security Evaluation”, Journal of Software, Vol.20, No. 2, pp. 451-461, Feb. 2009.

[6] Feng Dongqing, Sun Changfeng, and Fei Minrui, "Research on a New LMS Algorithm with Variable StePlength", Process Automation Instrumentation, Shanghai, china, Vol. 7, pp. 67-69, 2007. 